\title{
Overview of the Mechanism of Acupuncture and Moxibustion treatment of Decreased Ovarian Reserve
}

\author{
Mao Lin ${ }^{1}$, Tongsheng Su, ${ }^{1,2 *}$ \\ ${ }^{1}$ Shaanxi University of Traditional Chinese Medicine, Xianyang 712046, Shaanxi Province, China \\ ${ }^{2}$ Shaanxi Provincial Hospital of Traditional Chinese Medicine, Xi'an 710003, Shaanxi Province, China \\ *Corresponding author: Tongsheng Su, 811694167@qq.com
}

\begin{abstract}
Decreased ovarian reserve is one of the hot topics in current research. This article discusses the mechanism of acupuncture treatment of decreased ovarian reserve through the current acupuncture and moxibustion experimental research on the endocrine system and immune system.
\end{abstract}

Keywords: Acupuncture; Decreased ovarian reserve; Mechanism; Overview

Publication date: July 2021; Online publication: July 31, 2021

\section{Introduction}

Diminished Ovarian Reverse (DOR) is a reduction in the number of viable follicles remaining in the ovary, the decrease of oocyte quality, accompanied by high gonadotropin and low estrogen levels, causes the dysfunction of female reproductive endocrine function. Epidemiological studies have shown that the incidence of DOR is $10 \%{ }^{[1]}$. Western medicine in treatment mainly uses hormone replacement therapy, which has large side effects and many contraindications. In recent years, studies have shown that acupuncture and moxibustion have a significant effect on the treatment of this disease, and there is no toxic side effect. Here is a brief review of the mechanism of acupuncture and moxibustion in the treatment of decreased ovarian reserve.

\section{Mechanism research}

\subsection{Regulate the hypothalamus-pituitary-ovarian axis}

A complete and coordinated neuroendocrine system is formed between the hypothalamus, pituitary gland, and ovaries. Disorders in any of these links will cause ovarian dysfunction. Li You et al. ${ }^{[2]}$ found that electroacupuncture "Sanyinjiao" can improve natural perimenopausal periods. The level of estradiol (E2) in the ovary of rats reduces the level of follicle-stimulating hormone (FSH), luteinising hormone (LH) in the pituitary and Gonadotropin hormone-releasing hormone $(\mathrm{GnRH})$ in the hypothalamus. $\mathrm{Na} \mathrm{Li}^{[3]}$ found that counter-acupuncture and counter-moxibustion at the "Guanyuan" point can increase the levels of ER and GnRH in the hypothalamus and E2 and ER of the uterus in ovariectomized rats, and reduce the levels of FSH and LH in the pituitary gland. It shows that acupuncture has an overall and bidirectional regulating effect on the hypothalamic-pituitary-ovarian axis, and restores the balance of the abnormally functioning hypothalamic-pituitary-ovarian axis. 


\subsection{Regulating ovarian granulosa cell apoptosis}

Apoptosis is manifested as follicular atresia in the ovary, which is caused by the apoptosis of ovarian granulosa cells and oocytes ${ }^{[4]}$. Apoptosis involves a variety of factors. Studies have shown that ${ }^{[5]}$ the Bcl-2 family regulates the apoptosis of granulosa cells through the mitochondrial pathway, which plays a decisive role in the apoptosis of ovarian granulosa cells. In addition, death is caused by the activation of the Fas/Fas-L pathway. The receptor pathway is also one of the important factors of apoptosis ${ }^{[6]}$. The main components of the Bcl-2 family are Bcl-2 and Bax. An increase in the ratio of Bcl-2 inhibits apoptosis, and an increase in the ratio of Bax promotes apoptosis. Jiahong $\mathrm{Si}^{[7]}$ found that the Zhibian permeable channel acupuncture method can up-regulate Bcl-2 protein and down-regulate Bax protein, thereby inhibiting granulosa cell apoptosis. Xiao Ma ${ }^{[8]}$ found that acupuncture of Shenshu, Zusanli and Sanyinjiao could up-regulate the expression of Bcl-2 mRNA in perimenopausal rats. Down-regulating the expression of Fas mRNA in granulosa cells, Ma Xiaopeng ${ }^{[9]}$ and other studies found that acupuncture can down-regulate the expression of Fas protein and up-regulate the expression of Bcl-2 protein in ovarian granulosa cells. It can be seen that acupuncture regulates cell apoptosis by regulating protein expression on granulosa cells to inhibit follicular atresia and delay the decline of ovarian reserve.

\subsection{Inhibition of autoimmune response}

Many DOR patients have a variety of autoimmune antibodies in the blood [10], such as anti-nuclear antibodies, anti-zona pellucida antibodies, anti-ovarian antibodies, etc., which can cause autoimmune disorders, damage ovarian function, and reduce the number of follicles. Acupuncture has the dual effects of immunoprotection and immunosuppression. $\mathrm{Lu} \mathrm{Ge}{ }^{[11]}$ found that acupuncture on both sides of Shenshu, Guanyuan, and Zhongwan was used to treat rats with reduced ovarian reserve. The results showed that serum AMH, E2, and IL- 10 increased, FSH, IL-21 decreased, acupuncture therapy can inhibit the pro-inflammatory factors in the serum of rats with hypoovarian reserve, and can improve the level of sex hormones in rats and restore the estrous cycle of rats. Xiaoling Feng ${ }^{[12]}$ found that oral Yuyin pills combined with electroacupuncture on both sides of Sanyinjiao and Zusanli can improve the systemic symptoms, menstruation and serum sex hormone levels in patients with liver and kidney yin deficiency type ovarian reserve, and increase serum IL-4 and IL-10 level. Acupuncture can regulate the expression of cytokines, balance the pro-inflammatory and anti-inflammatory effects, improve the development of follicles, and restore ovarian function.

\section{Discussion}

In summary, acupuncture treatment of DOR is a multi-target, multi-channel benign regulatory effect, and there are no reports of adverse reactions and side effects. It is worthy of further promotion and application in clinical practice. But for the study of the mechanism of acupuncture treatment of DOR, at present, most of them are concentrated on one or a few targets of DOR, lacking systematic and holistic observation and evaluation. In recent years, some scholars have used advanced technology to explore the mechanism of acupuncture and moxibustion at the genetic level. Although it is not clear, it has multiple perspectives. In-depth research is the future direction of development.

\section{Disclosure statement}

The author declares no conflict of interest.

\section{References}

[1] Yang LK, Zhang JJ, Zhang ZZ, et al., 2019, The Effect of Acupuncture Chongren Mai on Sex 
Hormones in Patients with Decreased Ovarian Reserve. China Medical Herald, 16(24): 157-161.

[2] Li Y, Xu L, Qin Z, et al., 2014, The Effect of Electroacupuncture "Sanyinjiao" on the Hypothalamic-Pituitary-Ovarian Axis in Natural Perimenopausal Rats. Acupuncture Research, 39(03): $198-201$.

[3] Li N, Cheng K, Zhou J, 2013, Anti-acupuncture and Moxibustion of Guanyuan on the Hypothalamic-Pituitary-Ovarian Axis in Ovariectomized Rats. Chinese Journal of Information on Traditional Chinese Medicine, 20(08): 45-47.

[4] Shen H, 2012, Granular Cell and Oocyte Development. International Journal of Reproductive Health/Family Planning, 31(05):344-347.

[5] Cai L, Li L. Zhao YL, et al., 2020, Apoptosis Mechanism of Premature Ovarian Failure and Research Progress of Acupuncture Intervention. Journal of Reproductive Medicine, 29(03): 407-411.

[6] Lin PF, Hao YB, Guo HL, et al., 2010, The Effect of Fas/FasL on the Apoptosis of Granulosa Cells During In Vitro Culture of Isolated Porcine Follicles. Zoological Research, 31(03): 268-274.

[7] Si JH, et al., 2020, The Effect of Zhibian Permeable Channel Acupuncture on the Expression of Sex Hormones and Bcl-2 and Bax in Rats with Premature Ovarian Insufficiency. Shanxi University of Traditional Chinese Medicine.

[8] Ma XP, Dai M, Wu HG, et al., 2007, The Effect of Acupuncture on the Expression of Fas and Bcl-2 mRNA in Ovarian Granulosa Cells in Perimenopausal Rats. Shanghai Journal of Acupuncture and Moxibustion, (11): 35-38.

[9] Ma XP, Dai M, Wu HG, et al., 2007, The Effect of Acupuncture on Ovarian Granulosa Cell Apoptosis and Related Gene Expression in Perimenopausal Rats. Chinese Acupuncture and Moxibustion, (05): 357-361.

[10] Jia ZQ, Feng XL, Zhao Y, et al., 2019, Research Progress in the Treatment of Decreased Ovarian Reserve with Acupuncture and Medicine. Chinese Journal of Traditional Chinese Medicine, 34(03): 1124-1127.

[11] Lu G Xie ZJ, Wang Q, et al., 2020, The Effect of Acupuncture on Serum Inflammatory Factors and Ovarian Tissue Nrf2/HO-1/NLRP3 Signaling Pathway in a Rat Model of Ovarian Reserve Hypofunction. Journal of Traditional Chinese Medicine, 61 (15):1350-1356.

[12] Feng XL, Jia ZQ, Li N, et al., 2020, Effects of Electroacupuncture Combined with Yuyin Pills on Sex Hormones and Th2 Cytokines in Patients with Liver and Kidney Yin Deficiency Type Ovarian Reserve Decline. Chinese Acupuncture and Moxibustion, 40(09) :959-963. 